\title{
Correction to: AIDS Patient Care and STDs 2016;30:221-228; DOI: 10.1089/apc.2015.0333
}

TN The ARticle, "HIV Disclosure and Transmission Risks to Sex Partners Among HIVPositive Men," published in AIDS Patient Care and STDs 2016;30:221-228, there was an error in the reference list.

Reference 17, by K. Tassiopoulos et al., was cited, but the wrong Tassiopoulos et al. reference was provided in the reference list for the statement that "...rates of HIV disclosure to sex partners are similar to those observed among HIV-infected youth, where $67 \%$ report not disclosing their HIV status to first-time sex partners." The correct reference is as follows:

17. Tassiopoulos K, Moscicki AB, Mellins C, et al. Sexual risk behavior among youth with perinatal HIV infection in the United States: Predictors and implications for intervention development. Clin Infect Dis. 2013;56:283-90.

The authors thank Aliza Barnett, Senior Intern, Global Institute for Health and Human Rights, SUNY Albany, for noting this error. The online version of the article has been corrected to reflect this change.

The authors regret this error. 